\title{
Improved transport and culture system for the rapid diagnosis of gonorrhoea
}

\author{
A. E. JEPHCOTT*, M. N. BHATTACHARYYA ${ }^{\star}$, AND D. H. JACKSON† \\ Public Health Laboratory ${ }^{\star}$ and Royal Infirmary†, Sheffield
}

\section{Summary}

The Jembec/Neigon transport-and-culture system for the rapid diagnosis of gonorrhoea has been assessed by 167 sets of investigations in 128 named female contacts of infected men. The system offers the same advantages of efficiency and speed as the Transgrow transport-and-culture sysyem, but it is also more flexible and much less time-consuming.

\section{Introduction}

The Transgrow transport-and-culture system, when combined with fluorescent antibody (FA) identification, has been shown to reduce dramatically the time required for the cultural diagnosis of gonorrhoea (Jephcott, Morton, and Turner, 1974). Transgrow, described by Martin and Lester (1971), employs a selective culture medium layered inside a $\mathrm{CO}_{2}$ containing bottle. The method proved efficient but raised technical problems. Condensation combined with the curvature of the glass frequently so obscured the surface of the medium that daily fluorescent antibody examinations of samples obtained 'blindly' from almost every culture were essential for early diagnosis. For some laboratories this would represent an unacceptable work-load.

A new system-the Jembec/Neigon systemoffers easy direct access to the surface of the culture medium, so that an FA examination needs to be carried out only if suspicious bacterial growth has occurred.

This reduces the number of FA examinations to an acceptable level and makes the new method a practicable proposition for the average diagnostic laboratory.

The study reports a trial of commercially prepared Neigon plates (Flow Laboratories). The reliability of this transport-and-culture method and the speed with which it allows FA identification of gonococci has been compared with results using the conventional transport medium/delayed culture method.

\section{Material, methods, and patients}

The Jembec/Neigon system is a sophisticated development of the Bag- $\mathrm{CO}_{2}$-generating-tablet system described by Martin, Armstrong, and Smith (1974). It consists of a

Received for publication October 15, 1975

Address for reprints: Dr A. E. Jephcott, Public Health Laboratory, Northern General Hospital, Herries Road, Sheffield S5 7AU rectangular culture plate containing a selective gonococcal culture medium with vancomycin $3.5 \mathrm{mg}$./1., colistin $7.5 \mathrm{mg}$./1., trimethoprim $5.0 \mathrm{mg} . / 1$., and nystatin 50,000 $\mathrm{u} /$ litre. The plate incorporates a small recess into which a $\mathrm{CO}_{2}$-generating tablet is placed at the time of inoculation. With its well-fitting lid replaced, the plate is inserted into a plastic bag (described as an environmental pouch) which is sealed to make it gas-tight (See Figure). The moisture of the plate ensures $\mathrm{CO}_{2}$ generation due to reaction between the citric acid and sodium bicarbonate in the tablet. Plates were incubated immediately at $37^{\circ} \mathrm{C}$ with the base of the plate on the floor of the incubator. They were transported from the clinic to the supporting laboratory when convenient. Specimens
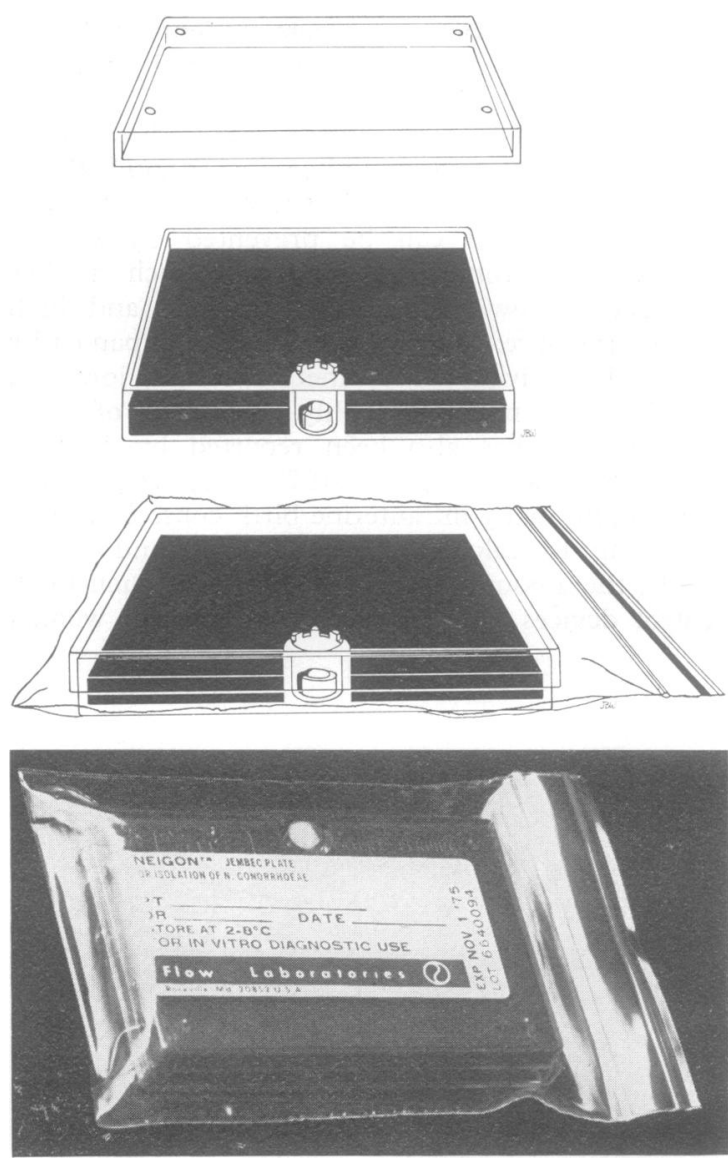

FIGURE Fembec plate and environmental pouch 
arrived at the laboratory within $24 \mathrm{hrs}$ except for those taken on Saturday mornings or before statutory holidays.

On arrival the plates were removed from their bags and the surface of the culture medium inspected for bacterial colonies. If these were detected they were tested for oxidase reaction, and smears were made for Gramstaining and FA testing using a method to be described. Plates showing no suspicious colonies were re-incubated and re-examined after a further 24 and $48 \mathrm{hrs}$.

Control culture specimens using identical duplicate swabs were taken in a carefully randomized order at the same time as those for the Jembec-Neigon system and were placed in Amies' transport medium. These accompanied the Jembec/Neigon plates to the laboratory for inoculation on to a non-selective Columbia chocolate agar medium and on to the selective medium described by Seth (1970).

Inoculated plates were incubated in a $\mathrm{CO}_{2}$ incubator for $48 \mathrm{hrs}$ before examination in the same manner as the Neigon plates.

The materials and methods and their reliability were first tested in a series of 59 male patients with a urethral discharge showing Gram-negative diplococci on initial Gram-staining. Thereafter 128 named female contacts of men with culture-proven gonorrhoea were tested. Each woman had randomized paired specimens taken from the urethra, endo-cervix, and rectum, on up to three separate occasions depending on the findings.

Similarly paired rectal specimens from eight passive male homosexuals were also tested.

Those patients selected represented the clinic's population in terms of age, marital, and social status.

\section{Results}

Table I shows the findings in the 59 men with urethritis; 58 were diagnosed as having gonorrhoea: 54 by both conventional and trial method, two by conventional means only, and two more by the trial system alone. Each man was tested on one occasion. The 59th man was found to have meningococcal urethritis.

Table II shows the time from taking the paired specimens to reporting positive results. For the conventional method the average time was 3.45 days (range 2 to 6 ) and for the Jembec/Neigon system the average time was 1.85 days (range 1 to 6 ). These times include weekends and statutory holidays.

TABLE II Time taken to report gonorrhoea culture results in 58 males

\begin{tabular}{|c|c|c|c|}
\hline \multicolumn{4}{|l|}{ Method } \\
\hline \multicolumn{2}{|c|}{ Conventional culture (days) } & \multicolumn{2}{|c|}{ fembec/Neigon (days) } \\
\hline Average & Range & Average & Range \\
\hline 3.45 & $2-6$ & 1.85 & $1-6$ \\
\hline
\end{tabular}

Table III shows the findings in the 128 named female contacts; 85 ( 66 per cent.) were diagnosed as having gonorrhoea. The 128 contacts had a total of 167 sets of diagnostic investigations. Repeat investigations were carried out on consecutive days as nearly as possible. Duplicate positive as well as duplicate negative investigations therefore occurred. 88 of the 167 sets of investigations gave positive results: 62 by both conventional and trial methods, eleven by conventional means only, and fifteen by the trial system only.

167 sets of investigations each provided three pairs of samples. Of these 501 pairs of samples, 269 gave positive results; 105 paired samples were positive by both conventional and trial methods; 24 by conventional delayed means only and a further 35 by the Jembec-Neigon system alone (see Table IV).

Table V shows that, for the conventional method, the average time from taking specimens to reporting positive findings was 3.37 days (range of 2 to 7) and for the Jembec-Neigon system 1.52 days (range 1 to 4$)$.

TABLE $\mathrm{V}$ Time taken to report positive gonorrhoea culture results of 167 sets of tests in 128 female contacts

Method

\begin{tabular}{|c|c|c|c|}
\hline \multicolumn{2}{|c|}{ Conventional culture (days) } & \multicolumn{2}{|c|}{ fembec (days) } \\
\hline Average & Range & Average & Range \\
\hline 3.37 & $2-7$ & 1.52 & $1-4$ \\
\hline
\end{tabular}

TABLE I Cultural diagnosis in 59 'smear positive' males

\begin{tabular}{|c|c|c|c|c|}
\hline \multirow[b]{2}{*}{ No. of patients } & \multicolumn{4}{|l|}{ No. positive } \\
\hline & Conventional method & fembec/Neigon method & Both methods & Either method \\
\hline $59^{a}$ & 56 & 56 & 54 & 58 \\
\hline
\end{tabular}

ancludes one case of meningococcal urethritis

TABLE II I Cultural findings using 167 pairs of investigations in 128 named female contacts

\begin{tabular}{|c|c|c|c|c|c|}
\hline \multirow[b]{2}{*}{$\begin{array}{l}\text { Total no. of pairs of } \\
\text { investigations }\end{array}$} & \multicolumn{5}{|l|}{ No. positive } \\
\hline & Conventional method & $\begin{array}{l}\text { Fembec/Neigon } \\
\text { method }\end{array}$ & Both methods & $\begin{array}{l}\text { Conventional } \\
\text { methods only }\end{array}$ & $\begin{array}{l}\text { Fembec/Neigon } \\
\text { only }\end{array}$ \\
\hline 167 & 73 & 77 & 62 & 11 & 15 \\
\hline
\end{tabular}


TABLE IV Cultural diagnosis in 128 female contacts, analysed by site demonstrated to be infected

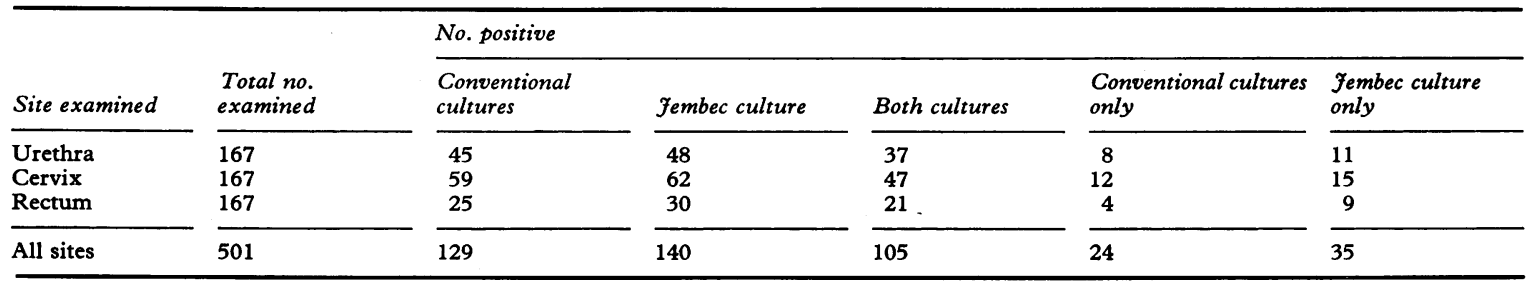

One homosexual of the eight tested gave positive results by both methods.

\section{Discussion}

Reducing the time for the cultural diagnosis of gonorrhoea not only benefits the individual patient but makes a worthwhile contribution to control of the disease. Use of the Transgrow transport-andculture system has been demonstrated as an effective way of matching these clinical and epidemiological needs (Jephcott and others, 1974). Speed was achieved by allowing bacterial growth to start immediately the patient had been examined and by identifying the growth within 24 to $48 \mathrm{hrs}$ using the rapid FA method. The disadvantages inherent in the Transgrow/FA system were technical. Many specimens were obtained "blindly" and FA examination was essential. These proved costly in terms of material and particularly in terms of manpower. These problems are readily overcome by the Jembec/Neigon system which allows direct access to the surface of the medium. Inspection and selection of colonies reduces the number of FA tests necessary to an acceptable and irreducible level. The trial shows that this has been achieved without significant loss of sensitivity or speed in obtaining a diagnosis.

Gonococcal culture systems employing only a selective medium will miss some infections due to antibiotic-sensitive strains (Reyn, 1973). Comparison of routine culture results in Sheffield on selective and non-selective media indicate that such antibioticsensitive strains represented almost one half of the strains of gonococci not detected by the Jembec/ Neigon system under trial. In practice however, direct plating and prompt incubation seem to have compensated for this.

There are many methods of detecting gonococci by culture. If the diagnosing laboratory is situated in the clinic then the Jembec system offers little advantage, apart from space saving, over conventional direct plating systems, but if transportation is essential then its flexibility can be a great advantage.

Certain laboratories employ 'presumptive gonococcus' diagnosis (Schroeter and Pazin, 1970), which is based on the demonstration of oxidasepositive colonies of Gram-negative diplococci on selective media, when the specimens come from genito-urinary sites. This lends itself well to the Jembec system. In fact, on only one occasion in this trial would such a diagnosis have proved incorrectwhen a urethral meningococcal infection would have been reported as positive by these criteria. However, the colonial appearance was very unlike that of a gonococcus and it is improbable that confusion would have arisen. The easy access to the colonies and the very high correlation with $\mathrm{FA}$ diagnosis must make this system very attractive to those less expensively equipped laboratories throughout the world where more complex tests are not possible. Similarly, certain laboratories may consider identification by sugar fermentation or sensitivity testing desirable. These can easily be performed because accurate removal of single bacterial colonies is possible. Such facilities are not available with Transgrow.

The Jembec-Neigon transport-and-culture system coupled with FA identification provides greater flexibility and fewer penalties than any of its predecessors. It is a reliable, speedy, and accurate method for the diagnosis of gonorrhoea. All the advantages of the earlier Transgrow system have been retained but the requirement for multiple FA examinations of every culture has been avoided, and ready access to bacterial colonies allows other tests to be conveniently performed.

We thank Miss D. Coles, Mr. B. Collins, Mr. W. E. Shaw and clinic staff for their invaluable assistance, and Dr. R. S. Morton for his advice and support. Flow laboratories provided a supply of Neigon plates.

\section{References}

Jephcott, A. E. Morton, R. S., and Turner, E. B., (1974) Lancet, 2, 1311

Martin, J. E., Armstrong, J. H., and Smith, P. B. (1974) Appl. Microbiol., 27, 802

- and Lester, A. (1971) Hlth Serv. ment. Hlth Admin. Rep., 86, 30

ReYN, A. (1973) Ugeskr. Laeg., 135, 909

Schroeter, A. L., and Pazin, G. J. (1970) Ann. intern. Med., 72, 553

Seth, A. (1970) Brit. F. vener. Dis., 46, 201 